\title{
Relación entre el bienestar social de Profesores y el Nivel de Autonomía y Tamaño de Escuelas Municipalizadas Chilena
}

\section{The Relation between Teachers` Social Well-being and the Level of Autonomy and Size of Chilean Public-Municipal Schools}

\author{
Paula Ascorra \\ Verónica López \\ M. Ángeles Bilbao \\ Trinidad Correa \\ Javier Guzmán \\ Valentina Moraga \\ Dayana Olavarría \\ Pontificia Universidad Católica de Valparaíso, Chile
}

Rec (19 de Marzo de 2013) Acept (20 de Mayo de 2014)

\section{Resumen}

El presente estudio tuvo por objetivo conocer el bienestar social en la escuela de profesores adscritos a establecimientos educacionales municipales que presentan niveles distintos de autonomía en su gestión administrativa (escuelas emergentes y autónomas) y distintos tamaños (escuelas grandes y pequeñas). Se trabajó con una muestra de 78 escuelas y 899 profesores distribuidos en cargos de directivos, docentes y asistentes. Los resultados globales indican que no existen diferencias significativas entre el bienestar social en la escuela de establecimientos autónomos y emergentes. No obstante, es mayor el nivel de bienestar social de los profesores adscritos a escuelas autónomas pequeñas. Por otro lado, los resultados avalan que una alta participación de los docentes en la gestión escolar está altamente relacionada con buenos niveles de bienestar social. Se discute que estas variables organizacionales -autonomía en la gestión, tamaño y participación de profesores- sí inciden en la salud mental de los profesores.

Palabras clave: bienestar social, bienestar, docentes, gestión escolar y tamaño.

\begin{abstract}
This purpose of this study was to characterize the social well-being of teachers working in public-municipal schools with different levels of autonomy in their school management (emergent and autonomous schools) and school size (large and small). 78 schools and 899 teachers working as school leaders, classroom teachers and teacher assistants, participated in this study. Results show no significant differences between teachers' global social well-being in autonomous versus emergent schools. However, the social well-being of teachers working in small autonomous schools was higher. Results also support the notion that a higher level of teacher participation in school management is associated with good levels of social well-being. We discuss that these organizational variables -autonomy in school management, school size, and teacher participation- have a significant impact on teachers' mental health.

Keywords: social well-being, well-being, teachers, school management, school size.
\end{abstract}

Correspondencia: Paula Escorra, Av. Brasil 1290, Sausalito, Viña del Mar, Chile. pascorra@ucv.cl

Este estudio fue financiado por el Proyecto FONDEF-IDEA CA12i10243 


\section{Introducción}

Se espera que el trabajo docente esté orientado por objetivos definidos

socialmente. Por lo tanto, las condiciones sociales de este trabajo son de enorme importancia para el logro de los objetivos pedagógicos, por un lado, y también para el bienestar y la salud de los propios docentes.

UNESCO 2005, p. 86.

Comprender el quehacer del profesorado desde una perspectiva del bienestar social implica aceptar que este bienestar emerge en un contexto particular y situado (Casas, 1996), en el cual se formulan una serie de necesidades, problemas y aspiraciones colectivas. No es posible pensar en el bienestar social sin considerar las condiciones y el contexto en el cual el individuo se desarrolla. En este sentido, el bienestar social toma en consideración al individuo y su contexto; el mundo dado y el mundo intersubjetivamente construido; las condiciones en que se desarrolla un trabajo y la percepción que realizan los individuos de ellas (Bang, 2014).

Siguiendo estas premisas, se hipotetiza que el nivel de bienestar social de profesores chilenos estará dado por las percepciones y las valoraciones que ellos realicen respecto de los procesos, circunstancias y contextos laborales en los que habitan y las condiciones organizacionales que las escuelas ofrezcan; entre ellas, autonomía, participación, apoyo, etc.

Vale la pena recordar que la implementación de la política educativa en nuestro país ha acarreado una enorme transformación del entorno laboral de los profesores y, por lo tanto, se esperarían cambios en su nivel de bienestar social. La perspectiva modernizante y tecnocrática (Redondo, 2002) que ha adoptado el Estado de Chile ha focalizado su quehacer en los procesos administrativos (estandarización, rendición de cuentas, tecnificación de oficios), en desmedro del ejercicio profesional, reflexivo y autónomo de los profesores (Assael, et al., 2011). De acuerdo a Robalino (2005), el modelo prescriptivo con el que opera la educación está socavando seriamente la autonomía profesional de los docentes, pues transforma a los profesores en burócratas que operan en la escuela ejecutando decisiones elaboradas en otras instancias.

Investigaciones internacionales demuestran que la autonomía está directamente asociada a la felicidad, a la construcción de un proyecto vital con sentido y a la percepción de control del entorno (Bilbao et al., 2011; Veliz-Burgos, 2012). A su vez, el tamaño de una organización constituye un aspecto fundamental para alcanzar metas comunes (Gil y Alcover de la Hera, 1999) y trae consigo una mayor satisfacción para los agentes educativos, ya que generan condiciones que facilitan el hacer comunidad (Prieto, 2001).

Atendiendo a lo anterior, la investigación que presentamos tuvo por propósito comparar los niveles de bienestar social de profesores que pertenecen a escuelas que poseen distinto grado de autonomía (escuelas emergentes y autónomas) y distinto tamaño (pequeñas y grandes según concentración de profesores). Nos parece sugerente asociar características de la gestión organizacional (grado de autonomía y tamaño) a la construcción del bienestar social de los profesores, puesto que de acuerdo a Keyes (1998), el bienestar social es simplemente la valoración que los sujetos realizan del funcionamiento y circunstancias de un contexto social.

Comprender las condiciones organizacionales en cuanto participantes del bienestar social de los profesores hace posible visibilizar que los cambios administrativos traen inextricablemente aparejados cambios en la subjetividad (Ascorra, Mandiola y Moya, 2011) y bienestar de los docentes (Pillay, Goddard y Wills, 2005). La consideración de este antecedente es importante, pues implica que la gestión administrativa y la política pública deben reflexionar y hacerse cargo de los fenómenos psicosociales que ellas acarrean.

\section{Bienestar social docente}

El concepto de bienestar en el ámbito de la psicología se viene desarrollando desde hace más de cuatro décadas (Diener, Suh, Lucas y Smith, 1999; Díaz y Sánchez, 2002) y en su devenir ha dado lugar a dos grandes tradiciones: La hedónica centrada en el bienestar subjetivo y la eudemónica centrada en el bienestar psicológico (Blanco y Díaz, 2005).

La tradición hedónica ha consolidado una reconocida línea de investigación que intenta responder "cómo y por qué la gente experimenta su vida de forma positiva, incluyendo tanto juicios cognitivos como reacciones afectivas" (Diener, 1994, p. 67). El bienestar subjetivo se inscribe en un marco principalmente afectivo y viene a dar cuenta de un estado general de satisfacción que construye la persona atendiendo a las oportunidades que le da la vida (capital social y cultural, recursos personales) y la experiencia emocional derivada de los acontecimientos que debe enfrentar en el desarrollo de ella (enfermedad, soledad, cesantía, opulencia, etc.).

Por otra parte, en la tradición eudemónica se desarrolla el concepto de bienestar psicológico, el cual ha centrado su interés en el estudio de las capacidades y el crecimiento personal. Cabe distinguir que -desde esta última perspectiva- el foco de análisis apunta a la actitud con que el sujeto 
afronta los retos vitales y las soluciones que desarrolla para conseguir las metas que se plantea (Blanco y Díaz, 2005).

Pese a que ambas tradiciones aluden a las condiciones sociales en las cuales se desarrolla una persona, éstas no abordan a cabalidad la relación entre el escenario social y el bienestar. A pesar de esto, las investigaciones anteriores poseen un valor histórico distintivo, ya que conformaron la base teórica para estudios posteriores en torno al fenómeno del bienestar social, asunto que nos convoca en este artículo (Blanco y Díaz, 2005).

El constructo de bienestar social fue propuesto inicialmente por Keyes (1998) para hacer referencia al vínculo entre bienestar y contexto situacional donde éste surge. Keyes operativiza el bienestar social como un constructo multidimensional, complejo y sostenido en el tiempo. Desde esta perspectiva, se caracteriza el estado emocional de una persona a partir de las relaciones interpersonales que entabla en una comunidad y de las valoraciones que construye frente a la misma. El Bienestar Social, entonces, es definido como "La valoración que hacemos de la propia circunstancia y el funcionamiento de la sociedad" (Keyes, 1998, p.112), y puede ser medido desde componentes afectivos y cognitivos, tanto en la vida privada como en la vida pública de las personas. Keyes (1998) plantea las siguientes dimensiones: integración social, aceptación social, contribución social, actualización social y coherencia social. Para tal efecto, el autor otorga una escala de información específica sobre cada componente, junto a un indicador global de bienestar social ${ }^{1}$.

En la última década se han intensificado las investigaciones en bienestar docente debido a las múltiples demandas que los profesores deben enfrentar, derivadas de las reformas educacionales que diversos países han implementado. Así, se ha establecido que el ejercicio docente ha complejizado su labor al requerir de constantes actualizaciones disciplinares, pedagógicas y de tecnologías; al tiempo de responder a demandas de estudiantes, equipo de gestión, apoderados y comunidad. Esto ha acarreado un incremento en el estrés

\footnotetext{
Dada la ventaja que posee la Escala de bienestar social de Keyes (1998) para trascender un interés puramente individual, ésta ha sido utilizada reiteradamente por investigaciones psicosociales pertenecientes a diversas disciplinas de las ciencias sociales: estudios sobre perfil psicosocial y bienestar (Zubieta, Fernández, Muratori, Barreiro y Mele, s/f), salud y bienestar (Díaz, Blanco, Horcajo y Valle, 2007), bienestar en contextos de violencia política y social (Amarís, Blanco, Madariaga, Díaz y Arciniégas, 2008), democracia y percepción de bienestar social (Laca, Augusto, Santana, Ochoa, Yunuen et. al, 2011) y derechos humanos (Páez, Beristain, González-Castro, Basabe y de Rivera, 2011).
}

de los docentes, deserción escolar y agotamiento (Pillay, Goddard y Wills, 2005; Bustos y Cornejo, 2014).

A nivel internacional, la investigación de Ewing y Smith (2003) informó que entre el $25 \%$ y el $40 \%$ de los docentes principiantes de países occidentales abandonan la enseñanza los primeros años de trabajo. La explicación que se da dice relación con la enorme sobrecarga que tienen los profesores actualmente y el poco apoyo personal que reciben, lo que se traduce en bajos niveles de bienestar. Singh y Billingsley (1996) encontraron que factores como el estrés, el agotamiento, la sobrecarga de trabajo y la insatisfacción en el empleo contribuyen al desgaste de los docentes, mientras que factores tales como el apoyo administrativo y expectativas razonables de rol, disminuyen las posibilidades de abandonar la labor de profesor y aumentan el bienestar en el docente. Respecto de la política pública internacional en materia de educación, Darling-Hammond (1995) sostiene que el contexto que ofrece el gobierno al potenciar la interacción de profesores, valorar el trabajo docente y aumentar la participación y autonomía en la escuela, presenta una relación positiva con la satisfacción laboral. En la misma línea, Certo y Fox (2002) indican que la satisfacción con el trabajo docente está asociada a las condiciones laborales y administrativas que presentan las escuelas. Desde Brasil, Felfeder (2006) realiza un profundo llamado a revisar las concepciones de autonomía y su vinculación con la gestión educativa. A juicio de la investigadora, el no respeto de la autonomía de las escuelas genera una educación abstracta y estandarizada que impide hacerse cargo de las situaciones reales y concretas que enfrentan los docentes en sus actividades diarias, exponiendo al profesorado a bajos niveles de bienestar social.

La variable tamaño de las organizaciones ha ido cobrando cada vez mayor importancia dado el actual escenario laboral, caracterizado por la presencia de organizaciones en red que trabajan a grandes dimensiones y en distintos países (Abarca, 2004). En su ya clásico artículo sobre la demografía organizacional, Pfeffer (1983) señaló que el tamaño y proporciones de los grupos condicionan la forma y la naturaleza de la interacción social, lo que a su vez afecta el bienestar psicológico y las actitudes de los trabajadores. Se postula que a mayor tamaño, menores son los niveles de bienestar. Siguiendo esta misma línea, Gil y Alcover de la Hera (1999) han postulado que el tamaño de las organizaciones determina la calidad y el tipo de vínculo laboral que se construye en el trabajo. De acuerdo a los autores, tamaños organizacionales pequeños facilitan el trabajo en equipo, la sinergia y cohesión del grupo. 


\section{Política pública y condiciones laborales asociadas a bienestar docente en Chile}

La actual política educacional se enmarca en un paradigma de corte neoliberal -con una perspectiva modernizante y tecnocrática-, donde el Estado ejerce un rol predominantemente subsidiario y privatizador (Redondo, 2002). Desde esta lógica predominan los mecanismos de mercado para mejorar la enseñanza y el aprendizaje, como la estandarización de procesos, la competitividad y la tecnificación de los oficios (Assaél, Guzmán y Contreras, 1993). Esta nueva concepción de educación ha demandando un nuevo ejercicio profesional y un nuevo rol docente. Este nuevo rol se caracteriza por adoptar una orientación técnico operativa en desmedro de una orientación reflexiva social (Núñez, 2002).

Este escenario grafica una evidente estandarización de los procesos educativos, donde la pedagogía se asemeja a la producción industrial, dejando "de ser relevantes los sujetos que constituyen el hecho pedagógico -estudiantes y docentes- erigiéndose como instrumentos centrales de la pedagogía, los métodos y técnicas para la enseñanza" (Donoso, 2004, p. 3). En la misma línea, Assaél (2009) señala que los agentes educativos se normalizan, de manera que los procesos pedagógicos resultan posibles de estandarizar. Así, los profesores "pueden ser intercambiados como si fueran piezas de una fábrica” (p. 7). Las políticas educativas actuales conciben a los profesores como aplicadores de una serie de procedimientos diseñados en un ámbito ajeno a la escuela (Robalino, 2005). Es posible observar la imposición cada vez más frecuente de paquetes curriculares, guías didácticas e intervenciones que vienen empaquetadas y listas para ser aplicadas (prepackage manualized intervention programs). A juicio de Robalino (2005), estas prácticas impositivas desconocen e invalidan las construcciones elaboradas por los profesores y socavan seriamente su autonomía profesional convirtiéndolas en agentes pasivos.

Dentro de este marco de gestión se pone en ejercicio, el año 2008, la Ley de Subvención Escolar Preferencial ${ }^{2}$. Esta ley tiene el propósito de mejorar la calidad de la educación, otorgando igualdad de oportunidades a todos los niños y niñas del país. Para tal efecto, el Estado otorga a los establecimientos recursos diferenciados según el nivel socioeconómico del alumno, identificando a alumnos prioritarios ${ }^{3}$.

\footnotetext{
Esta Ley se aplica de manera obligatoria en la educación municipal. Las escuelas particulares subvencionados pueden acceder a ella de manera voluntaria.

3 Se entiende por calidad de prioritario -según criterios otorgada por el Estado chileno- a los estudiantes que posean una situación económica precaria, la cual es determinada anualmente en base a una forma de cál-
}

La ley clasifica a las escuelas de acuerdo a sus resultados en la prueba estandarizada SIMCE. Las escuelas catalogadas como autónomas son aquellas que han demostrado, sistemáticamente, buenos resultados educativos. Las instituciones categorizadas como emergentes no han mostrado buenos resultados de manera sistemática y aquellas clasificadas en recuperación han obtenido reiteradamente resultados deficientes. Las escuelas, al comenzar el régimen de la Ley SEP, se subscriben al Convenio de Igualdad de Oportunidades y Excelencia Educativa, lo que implica que cada escuela se compromete a llevar a cabo un Plan de Mejoramiento Educativo (PME), el cual será supervisado y evaluado por el ministerio. Aquellas escuelas que no cumplan su PME arriesgan ser bajadas de categoría o lisa y llanamente cerradas. La Tabla 1 presenta las principales diferencias en el grado de autonomía que se les asigna a los establecimientos según categorización SEP ${ }^{4}$.

La forma en que se ha implementado la ley y las diferencias que plantea entre categorías de escuelas, ha sido ampliamente cuestionada debido a los efectos que tendría sobre el bienestar social, la calidad de vida y la salud física y mental del cuerpo de profesores. En defensa de la autonomía, Assaél et al. (2011) sostienen que cada establecimiento educativo desarrolla significaciones propias acerca de su autonomía institucional, que propician o desarticulan espacios de diálogos, prácticas pedagógicas y modos particulares de ser una comunidad educativa. Intervenir este espacio relacional repercute en los niveles de bienestar social escolar, en el clima educacional y en la consolidación de equipos directivos.

Algunos autores han señalado la pérdida de autonomía como un producto histórico, distintivo de este cambio en la educación, y que puede ser traducido como un "continuo de enajenación" (Cornejo, 2007). Esto refiere a un menor grado de control y decisión sobre las prácticas educativas, lo que se manifiesta en una considerable baja en la sensación de autonomía de los docentes y una consecuente disminución de bienestar social asociado al trabajo (Cornejo, 2007).

A nivel de bienestar y salud mental de los docentes en Chile, Cornejo (2008) presenta alarmantes cifras que

culo que considera: los ingresos familiares, la escolaridad de los padres o apoderados, el grado de pobreza de su residencia, la pertenencia de sus familias al Programa Chile Solidario, posicionarse en el tercio más vulnerable según la Ficha de Protección Social, tener padres o apoderados ubicados en el tramo A del Fondo Nacional de Salud (FONASA) y la condición de ruralidad de su comuna. Las escuelas ingresadas a SEP reciben un monto de $\$ 19.980$ por estudiante. www.mineduc.cl

4 Elaboración propia, fuente "Un camino para la equidad: Ley de Subvención Preferencial”, Ministerio de Educación, p. 6. 
Tabla 1. Requerimientos de la Ley SEP Por categoría de escuela.

\begin{tabular}{ll}
\hline Autónomas & \multicolumn{1}{c}{ Plan Mejora Escolar (PME) } \\
& $\begin{array}{l}\text { El PME no requiere de la aprobación por parte del Ministerio. } \\
\text { según los resultados SIMCE. }\end{array}$ \\
& En caso de descender sus resultados, la escuela no arriesga el cierre \\
Emergentes & El PME debe responder a una estructura determinada por el Ministerio: diagnóstico con evaluación de recursos hu- \\
y en & manos, técnicos y materiales; metas de resultados educativos; iniciativas orientadas a coordinar y articular acciones \\
Recuperación & con las instituciones y redes de servicios sociales competentes para detectar, derivar y tratar problemas psicológicos, \\
& sociales y necesidades educativas especiales de los niños y niñas caracterizados como prioritarios, y establecer acti- \\
& vidades docentes complementarias a los procesos de enseñanza y aprendizaje de dichos alumnos. \\
& El PME debe ser aprobado por el Ministerio. \\
& En caso de descender sus resultados, la escuela arriesga el cierre \\
\hline
\end{tabular}

Fuente: elaboración propia.

denuncian un crecimiento de episodios de depresión mayor y trastornos ansiosos. A nivel general, han sido descritos como factores que ha sufrido el ejercicio docente la ambigüedad y conflictos en el rol de los profesores, la mantención de la disciplina, la desmotivación de los estudiantes, la falta de materiales de apoyo para el trabajo, las presiones de tiempo, el exceso de trabajo administrativo, el descenso en la valoración social de la profesión docente, la pérdida de control y autonomía sobre el trabajo y la falta de apoyo entre colegas (Cornejo, 2007).

Por consiguiente, en este estudio pusimos a prueba la hipótesis de que un contexto organizacional caracterizado por la autonomía en la gestión escolar, afectaría positivamente el nivel de bienestar social de docentes, directivos y asistentes de la educación. Considerando que los directivos presentan, además, un nivel de autonomía y participación mayor en la toma de decisiones de la escuela que docentes, y éstos a su vez mayor que asistentes de la educación, hipotetizamos que el nivel de bienestar social en la escuela sería mayor para directivos y menor para asistentes de la educación. Finalmente, dado que algunos estudios señalan que el tamaño de la escuela afecta la calidad y tipo de relaciones que en ella se dan, incorporamos el tamaño de la escuela como una variable de confusión.

\section{Método}

Esta investigación corresponde a un estudio comparativo de tipo cuantitativo, con un diseño de investigación no experimental. Se estableció una muestra representativa estratificada $^{5}$ de la Región de Valparaíso.

\footnotetext{
5 La muestra fue extraída a partir de la Base de Datos 2010, otorgada por la SEREMI de educación. Cabe señalar que a tal fecha, la Ley SEP
}

\section{Participantes}

Se determinó que la población la conformarían la cantidad de establecimientos municipales de la Región de Valparaíso adscritos a la Ley SEP. La unidad de análisis fueron las escuelas conformadas por docentes que realizaban diversas funciones: docentes ( $\mathrm{n}=734 ; 82 \%)$, asistentes de la educación $(\mathrm{n}=55 ; 6 \%)$ y directivos $(\mathrm{n}=110 ; 12 \%)$.

La estratificación de la muestra se estableció con afijación proporcional, utilizando dos criterios: tamaño (escuelas con 25 profesores o menos serían pequeñas y aquellas con 26 profesores o más serían grandes) y categorización SEP (al momento del trabajo en terreno del estudio nos brindaba dos categorías: autónomo y emergente). La muestra final alcanzó 78 establecimientos (ver Tabla 2).

Tabla 2. Establecimientos según clasificación SEP y tamaño.

\begin{tabular}{lcc}
\hline & Clasificación del Establecimiento \\
Tipo de Establecimiento & Autónomo & Emergente \\
\hline Pequeño & 17 & 48 \\
Grande & 5 & 8 \\
Total & 22 & 56 \\
\hline
\end{tabular}

\section{Instrumentos}

Escala de bienestar social en la escuela. Se trata de la adaptación de la Escala de bienestar social de Keyes (1998), según ha sido adaptada al español por Blanco y Díaz (2005). La adaptación se realizó con el objetivo de contar con una medida de bienestar social en la escuela, y consistió en modificar los términos referidos a la "sociedad" por "en esta escuela". El instrumento adaptado consta de 27 ítems estructurados en escala Likert, con opciones que van desde 1 (muy en desacuerdo) hasta 5 (muy de acuerdo). El instrumento presenta una adecuada consistencia interna de

estaba implementada sólo a nivel de escuelas básicas. 
0.94 y una estructura factorial de 5 factores (ver Tabla 3), que en conjunto explican el $60 \%$ de la varianza.

Tabla 3. Dimensiones de la Escala de bienestar social para docentes.

\begin{tabular}{|c|c|}
\hline Dimensión & Descripción \\
\hline Integración Social & $\begin{array}{l}\text { Ítems: } 1,2,3,4, y 7 \text {. Mide el grado en que } \\
\text { las personas consideran que tienen algo en } \\
\text { común con los miembros de su escuela y } \\
\text { el sentimiento de pertenencia a sus comu- } \\
\text { nidades educativas. }\end{array}$ \\
\hline
\end{tabular}

Aceptación Social İtems: 8, 9, 10, 11, 12, 13 y 14. Es la evaluación de los miembros de la comunidad educativa en términos de cualidades positivas, y sentirse aceptados por ellos.

Contribución Social Ítems: 16, 20 y 21. Creencia de que uno es un miembro vital de la escuela, refleja en qué grado la gente siente que lo que hace es un aporte valorado por su comunidad educativa.

Actualización Ítems: 23, 24, 26 y27. Evaluación del potenSocial cial de la escuela, creencia que se evoluciona hacia un futuro mejor. Implica si las personas tienen esperanza sobre las condiciones y el futuro de su comunidad escolar.

Coherencia Social Ítems: 17, 18, 19, 28, 29, 30, 31 y 32. Evalúa la comprensión de la dinámica de la comunidad escolar, así como la sensación de poder influir en la escuela.

Nota*. Se conserva el orden numérico de la Escala de bienestar social de la adaptación española de Blanco y Díaz (2005).

Cuestionario de Transferencia de la Ley Sep (TLS). Este instrumento construido ad hoc para este estudio, evalúa la implementación de la Ley SEP en los establecimientos educacionales. Se construyó de forma diferenciada para el equipo directivo y el plantel docente. El cuestionario para directivos contiene 9 preguntas con opciones de respuesta sí o no, seguido de 2 preguntas abiertas. El cuestionario refiere a temas como la autonomía en la toma de decisiones respecto de la utilización de recursos por concepto de Ley SEP; la autonomía para realizar contrataciones con recursos de la Ley SEP a docentes y profesionales psicosociales; la posibilidad de alcanzar las metas establecidas bajo la Ley SEP; repercusiones de esa consecución de metas; la autonomía para trabajar con una Asesoría Técnica Educativa (ATE) y decidir cuál. El análisis factorial de estos reactivos indicó una estructura de dos factores: autonomía y expectativas. En el apartado de preguntas abiertas, se consultó si estaba de acuerdo con la categorización SEP de su establecimiento y por qué, además de si consideraba que la gestión del establecimiento era autónoma, justificando por qué. Estas preguntas abiertas sirvieron de insumo para corroborar la clasificación de las escuelas en autónomas o emergentes, al tomar en cuenta, además de la clasificación oficial, la percepción subjetiva del nivel de autonomía de la escuela.

El cuestionario para docentes ${ }^{6}$ contiene 7 preguntas con opciones de respuesta sí o no, y luego una pregunta abierta. Refiere a la disponibilidad de horas no lectivas remuneradas para planificar; participación en diseño, implementación y evaluación del Plan de Mejoramiento Educativo (PME); realización de adaptaciones metodológicas; la posibilidad de alcanzar las metas establecidas por la Ley SEP y repercusión de la consecución de dichas metas. El análisis factorial de estos reactivos indicó una estructura de dos factores: participación y expectativas. En el apartado de preguntas abiertas se consultó si estaba de acuerdo con la categorización SEP que presentaba el establecimiento y por qué.

\section{Procedimiento}

Los establecimientos educacionales participaron voluntariamente del estudio, por medio de un consentimiento informado de parte de cada director, habiéndoles explicado en forma oral y por escrito los objetivos y aspectos generales de la investigación.

Para proteger la confidencialidad de las respuestas, cada establecimiento recibió la cantidad de encuestas correspondientes para el plantel docente y el equipo directivo de cada escuela. Las encuestas venían en sobres cerrados que se entregaron a los directores, para que ellos las repartieran y posteriormente recolectaran una vez respondidas. Este proceso demoró aproximadamente una semana. El tiempo estimado necesario para la aplicación de las encuestas fue de 15 minutos.

\section{Análisis de datos}

Para el análisis de resultados se utilizó el programa estadístico SPSS 15.0 para el cruce de las variables de interés, se utilizaron ANOVAS de un factor.

En el caso de la pregunta abierta que forma parte del cuestionario TLS, realizamos un análisis de tendencia de

\footnotetext{
6 Las preguntas que contempla el cuestionario son las siguientes: ¿Usted dispone de horas no lectivas remuneradas destinadas a tareas de planificación? ¿Usted participó en la elaboración del plan de mejoramiento educativo de su establecimiento? ¿Usted participó en la implementación del plan de mejoramiento educativo de su establecimiento? ¿Usted participó en la evaluación del plan de mejoramiento educativo de su establecimiento?¿Realiza adaptaciones metodológicas en función de necesidades educativas específicas detectadas por usted en el aula? ¿Usted cree que su escuela alcanzará las metas establecidas bajo la Ley SEP? ¿Cree usted que la consecución de estas metas aportarán a generar un futuro más auspicioso para su institución educativa?
} 
Tabla 4. Estadísticos descriptivos de la Escala de bienestar social, en la escuela para equipo escolar.

\begin{tabular}{lccccccccccccccc}
\hline & \multicolumn{4}{c}{ Autónomas } & \multicolumn{4}{c}{ Emergentes } \\
& \multicolumn{3}{c}{ Grandes } & \multicolumn{3}{c}{ Pequeñas } & \multicolumn{3}{c}{ Grandes } & \multicolumn{3}{c}{ Pequeñas } \\
\hline Integración social & 73 & 3.97 & 0.59 & 169 & 4.34 & 0.55 & 107 & 4.31 & 0.94 & 546 & 4.21 & 0.64 \\
Aceptación social & 75 & 3.46 & 0.96 & 168 & 4.02 & 0.75 & 107 & 3.84 & 0.58 & 547 & 3.83 & 0.84 \\
Actualización social & 75 & 3.68 & 0.72 & 169 & 4.13 & 0.79 & 107 & 3.98 & 0.82 & 545 & 3.90 & 0.80 \\
Contribución social & 75 & 4.38 & 0.63 & 169 & 4.43 & 0.68 & 107 & 4.56 & 0.49 & 547 & 4.43 & 0.58 \\
Coherencia & 75 & 4.15 & 0.84 & 168 & 4.49 & 0.64 & 107 & 4.16 & 1.14 & 541 & 4.27 & 0.80 \\
\hline Bienestar global & 73 & 3.90 & 0.59 & 167 & 4.28 & 0.53 & 107 & 4.12 & 0.69 & 535 & 4.11 & 0.59 \\
\hline
\end{tabular}

respuestas, que permitió agrupar los resultados obtenidos, estableciendo categorías.

\section{Resultados}

Los estadísticos descriptivos muestran que las medias generales oscilan entre 3.84 y 4.44 , lo que indica una inclinación positiva de los resultados del bienestar social en la escuela. Las dimensiones con medias más elevadas son las de contribución social y coherencia social escolar. La Tabla 4 presenta los estadísticos descriptivos de las dimensiones de bienestar social en la escuela.

Para comparar los niveles de bienestar social en la escuela, según la categoría SEP de los establecimientos, se utilizó la prueba $t$ de Student. Los resultados indican que en el bienestar social en la escuela global no hay diferencias significativas entre escuelas autónomas $(\mathrm{M}=4.17, D T=0.58)$ y emergentes $(\mathrm{M}=4.11, D T=0.61 ; t(880)=1.22 p=.22)$. El análisis por dimensión arrojó que sólo hay diferencias en la dimensión de coherencia, donde las escuelas autónomas presentaron mayores niveles $(\mathrm{M}=4.38 . D T=0.73)$ que las escuelas emergentes $(\mathrm{M}=4.25, D T=0.86 ; t(889)=2.03$, $p<.05)$. Esto revela que los docentes, directivos y asistentes de la educación pertenecientes a escuelas autónomas, tienen una mayor capacidad para entender el funcionamiento de su establecimiento que aquellos miembros que se desempeñan en escuelas emergentes. Esto es relevante ya que este dato puede estar asociado a la limitación en la autonomía pedagógica que impone la Ley SEP a las escuelas clasificadas como emergentes.

Atendiendo a que no se encontraron diferencias significativas en la escala global, se desarrolló una nueva variable codificando la categoría SEP y el tamaño de las escuelas, obteniendo 4 categorías, por ejemplo escuelas emergentes grandes. El objetivo era incorporar el tamaño de la escuela como variable de confusión (confounding variable) en la relación entre autonomía en la gestión escolar y bienestar social en la escuela. Se realizó una ANOVA entre bienestar social en la escuela con la variable categoría SEP-Tamaño.

Los resultados indican que el bienestar social global en las escuelas autónomas pequeñas es bastante mayor $(\mathrm{M}=4.28$. $D T=0.53)$ que el nivel de bienestar social en las escuelas emergentes pequeñas $(\mathrm{M}=4.11 . D T=0.59)$ y que las escuelas autónomas grandes $(\mathrm{M}=3.90, D T=0.59)(F(3,878)=746$, $p<.001)$. Cabe precisar que esta última categoría presenta los niveles más bajos de bienestar social en la escuela, particularmente en las dimensiones de integración social, aceptación social y coherencia social.

Estos resultados sugieren que los actores educativos pertenecientes a escuelas autónomas pequeñas mantienen vínculos interpersonales de calidad y se sienten aceptados por su comunidad, desarrollando un sentido de pertenencia con su escuela, mostrándose comprometidos con lo que sucede en ella. Las nociones señaladas no se aplican en escuelas autónomas grandes, quienes presentan una posición significativamente descendida en comparación con el resto de las categorías no hubo diferencias notorias entre escuelas emergentes pequeñas y grandes.

La prueba ANOVA de un factor indicó, según lo esperado, que los directivos presentan niveles más altos de bienestar social en la escuela, tanto en la escala global $(F(2,784)$ $=5.59, p<.01$ ) como en todas las dimensiones, menos en coherencia social (por dimensión: aceptación $F(2,889)=3.91$, $p<.05$; integración $F(2,887)=8.46, p<.001$; actualización $F(2,888)=3.24, p<.05$; contribución $F(2,890)=3.26, p<.05$; coherencia $F(2,883)=1.97, p=.14)$. Estos resultados reflejan que los directivos son los agentes educativos con mayor sentido de pertenencia y de aceptación de su comunidad escolar, lo que los mantiene comprometidos con los demás, realizando aportes que contribuyen al mejoramiento de la escuela y generando una disposición positiva hacia el futuro, pues creen en el potencial de su establecimiento. Por el contrario, los asistentes de la 

Javier Guzmán, Valentina Moraga y Dayana Olavarría

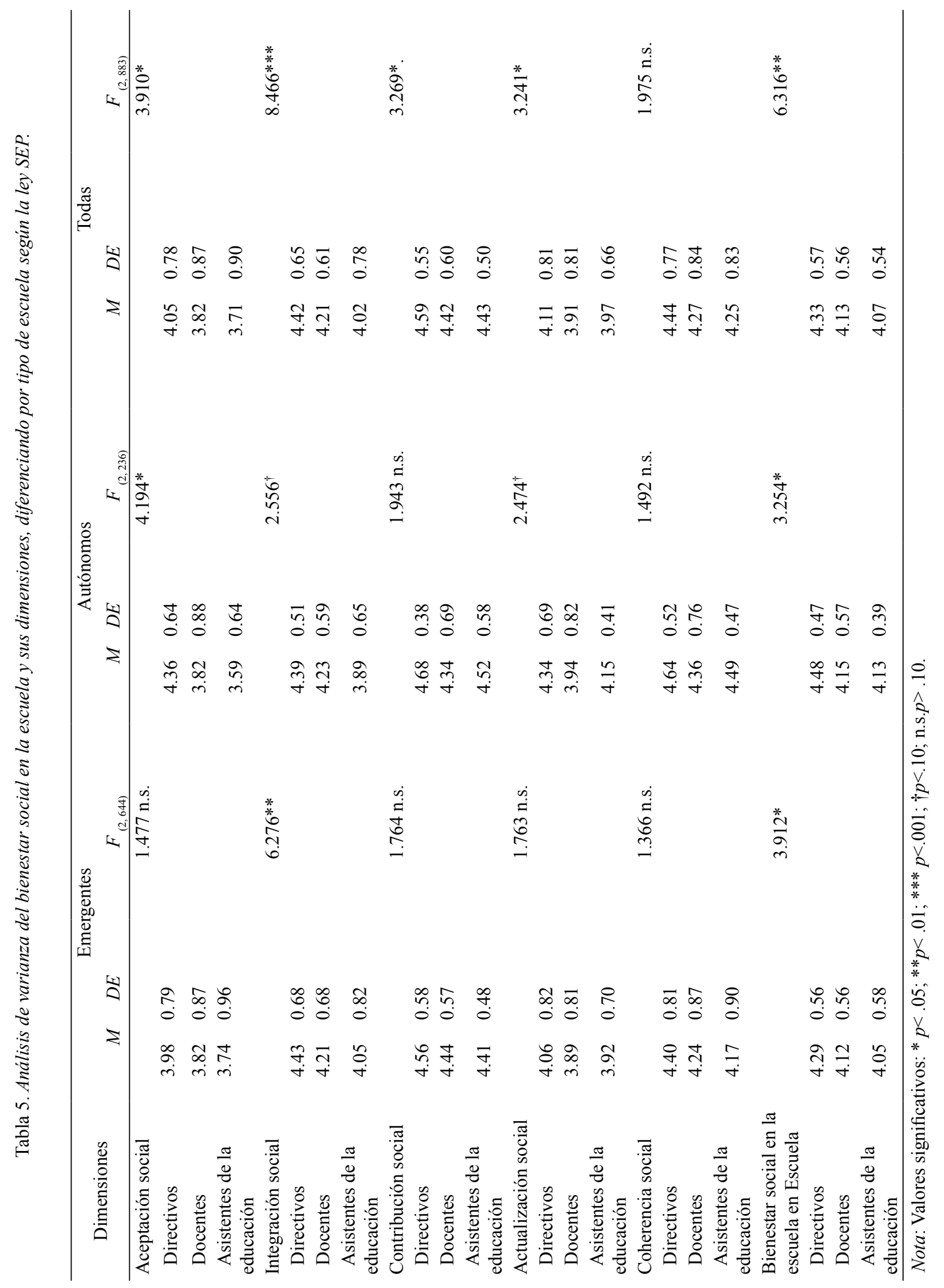


educación muestran sentirse menos aceptados y valorados (ver Tabla 5).

En relación al cuestionario TLS, las escuelas pequeñas autónomas presentan altos niveles de participación, con un $58.9 \%$ de docentes que participan en todas las actividades del PME y un $17.8 \%$ que participan en algunas de ellas. Quizás ésta sea una de las variables que explique mejor el por qué las escuelas autónomas pequeñas presentan los niveles más altos de bienestar social.

Los resultados comparativos entre escuelas autónomas y emergentes en el índice de participación respaldan lo anterior. Tanto en escuelas autónomas como emergentes, quienes participan en todas las actividades del PME presentan un mayor nivel de bienestar social que quienes participan en algunas actividades, o no participan. A su vez, cabe destacar que los docentes de escuelas emergentes que no participan poseen un bienestar social en la escuela considerablemente más bajo que los profesores que no participan en las escuelas autónomas $(M=3.86 . D T=0.68$, versus $M=4.10 . D T=0.58)$. Quienes participan en todas las actividades referidas del PME manifiestan niveles similares de bienestar social en la escuela, independientemente de la categoría SEP del establecimiento (ver Tabla 6).

\section{Discusión}

A la luz de los resultados obtenidos en la Escala de bienestar social en la escuela, podemos apreciar que los agentes educativos -en general- se sienten a gusto dentro de su comunidad escolar, pues los actores educativos encuestados se consideran un aporte para su escuela y sienten que son valorados por los miembros de dicha comunidad, comprendiendo la lógica de organización y funcionamiento de su propia institución. A su vez, creen que la educación en Chile está en un proceso de mejora y que la escuela a la cual pertenecen se encuentra en continuo desarrollo. Estos hallazgos contrastan con los planteamientos de Assaél et al. (2011) ya enunciados y con las investigaciones realizadas por Cornejo (2007), que evidencian altos índices de ansiedad y depresión en los profesores. Es relevante discutir este punto, pues si bien en términos individuales los profesores se sienten deprimidos y agotados, su percepción respecto del ambiente social en el cual se desarrollan sería buena.

En el contexto escolar, el tamaño de un grupo resulta ser un factor modelador de las variables psicoafectivas generadas al interior del mismo. Así, las escuelas pequeñas demostraron tener un mayor bienestar social que las escuelas grandes, lo cual nos confirma que las relaciones interpersonales constituyen un aspecto fundamental para alcanzar metas comunes (Gil y Alcover de la Hera, 1999). Esto trae consigo una mayor satisfacción para los agentes educativos, ya que existen objetivos compartidos por todos, generando espacios para hacer comunidad (Prieto, 2001), pues aquellos son grupos más cohesionados, lo que facilita el trabajo en equipo y sinergia de recursos para lograr metas (Gil y Alcover de la Hera, 1999). Cabe precisar que todos los elementos mencionados forman parte del concepto de Bienestar Social.

Destacamos que nuestra hipótesis de que existirían diferencias en el bienestar social entre las escuelas autónomas y emergentes -entendiendo su diferencia en los grados de autonomía institucional- se cumple parcialmente. Por una parte, los profesionales de las escuelas autónomas no presentan un nivel mayor de bienestar social global, aunque sí en la dimensión coherencia. Por otra parte, la combinación

Tabla 6. Resultados de análisis de varianza en bienestar social en la escuela en la escuela por grado de participación, comparación entre escuelas emergentes y autónomas.

\begin{tabular}{llllll}
\hline Variable & \multicolumn{2}{l}{ Emergentes } & Autónomos & Todas & \\
& $M(D E)$ & $F_{(2.514)}$ & $M(D E) F_{(2.205)}$ & $M(D E)$ & $F_{(2.722)}$ \\
\hline Participación & & & & \\
No & $3.86(.59)$ & $12.197^{* * *}$ & $4.12(.56) 4.654^{* *}$ & $3.96(.59)$ & $13.722^{* * *}$ \\
Relativa & $4.07(.53)$ & $3.93(.63)$ & $4.04(.56)$ & \\
Sí & $4.22(.57)$ & $4.22(.52)$ & $4.22(.55)$ & \\
\hline
\end{tabular}

Nota: Valores significativos $* * p<.01 ; * * * p<.001$. 
de las categorías tipo de gestión (autónoma/emergente) y tamaño de escuela (grandes/pequeñas) resultó relevante, pues las escuelas autónomas pequeñas presentan los mejores niveles de bienestar social, tanto para la escala global como en tres de las cinco dimensiones (integración social, aceptación social y coherencia). ¿Por qué los docentes de escuelas pequeñas y con gestión autónoma presentan mejores niveles de bienestar social? Los resultados del cuestionario TLS entregan luces sugerentes. Se puede apreciar que los directivos de escuelas autónomas poseen un mayor nivel de aceptación social y en términos relativos todas las otras dimensiones muestran altos niveles de bienestar. Ahora bien, tanto en escuelas autónomas como emergentes, los docentes que señalan participar más, presentan mejores niveles de bienestar social que quienes señalan no participar. Estos resultados sugieren que la participación es un elemento base en la construcción del bienestar social en la escuela. Esto nos permite vislumbrar un nuevo enfoque de análisis al interior de la comunidad, que alude a la participación de los actores educativos -hoy, en el diseño, implementación y evaluación del PME; mañana, en el nuevo instrumento de la política educativa- como un factor primordial sobre el que trabajar para mejorar el bienestar social de un equipo escolar. La participación facilita diversos procesos en la escuela: construye la dinámica organizacional, posibilita la contextualización de las políticas públicas a comunidades particulares, permite la autonomía - a nivel de aula e institucional-y potencia la construcción de sentidos comunes que orientan los objetivos de las escuelas (Prieto, 2011). Este planteamiento podría iluminar la política pública en educación en el sentido de otorgar mayor participación y autonomía a las escuelas clasificadas como emergentes y en recuperación, con el objeto de mejorar el bienestar social y la salud mental de los profesores. A su vez, sería pertinente dar mayor participación a los asistentes de la educación, quienes presentan los niveles más bajos de bienestar social.

Destacamos que, aunque las categorías de escuelas emergentes y autónomas son marcos conceptuales designados por el Ministerio de Educación, en nuestra investigación, variables como la participación en el diseño, implementación, evaluación del PME y el tamaño de la escuela son variables que nos permiten describir una autonomía al interior de la escuela, y son estas variables las que marcan la diferencia en el bienestar social de los equipos escolares. Por lo tanto, consideramos relevante observar las variables que son propias de las escuelas a nivel local, ya que son estos elementos los que nos permiten describir cómo funcionan y son realmente los establecimientos educativos (López y Ascorra, 2012).
Así, comprendemos el bienestar social en una perspectiva situacional y contextualizada (Casas, 1996).

Concluimos que sí existe una relación positiva entre el bienestar social medido individualmente y las características de los contextos de trabajo. Tal como lo sugiere la teoría, son las escuelas pequeñas, donde hay mayor grado de participación y autonomía, las que reportan los mejores niveles de bienestar social. Este llamado permite comenzar a considerar las variables institucionales como generadoras de salud, lo que se conoce como organizaciones positivas (Park, Peterson y Sun, 2013).

Dado que la presente investigación constituye un primer abordaje de bienestar social en el contexto educacional, se sugiere realizar estudios que se interesen por indagar en el bienestar social del resto de la comunidad escolar, como alumnos, auxiliares y apoderados. También es necesario ahondar cualitativamente en las atribuciones de los miembros de la comunidad respecto de los factores que pueden implicar mayor o menor bienestar social y su relación con otras variables asociadas a los contextos de trabajo, como puede ser el tipo de jornada laboral de los docentes, lo que es relevante en contextos de precarización laboral (Sisto y Fardella, 2011) y alta rotación docente en el sistema escolar chileno.

\section{Referencias}

Abarca, N. (2004). Inteligencia emocional en el liderazgo. Santiago de Chile: Aguilar.

Abello, R., Amarís, M., Blanco, A., Madariaga, C., Díaz, D., y Arciniégas, T. (2008). Bienestar, autoestima, depresión y anomia en personas que no han sido víctimas de violencia política y social. Investigación y Desarrollo, 2, 214-231, Colombia: Universidad del Norte.

Ascorra, P; Mandiola, M. y Moya, I. (2011). Modelos de gestión en Chile y la constitución del trabajador resignado. En A. Bilbao (Coord.) .Creación, identidad y mundo en los estados de la globalización (pp. 285-303). Valparaíso: Ediciones Universitarias.

Assaél, J., Guzmán, I., Contreras, M. (1993). Qué entendemos por calidad de la educación. Momento Informático, Boletín 18 T.E.D. PIIE. Santiago de Chile.

Assaél, J. (2009). Políticas educativas de estandarización y control: Su efecto en la gestión democrática y calidad de la enseñanza en educación. III Congreso Interamericano / XXIV Simposium Brasileño de Política de Administración de la Educación: Brasil.

Assaél, J., Budnik, J., Cornejo, R., Chávez, R., González López, J., Redondo, JM., Rojo, J., Sánchez Edmonson, R..y Sobarzo-Morales, M. (2011). La empresa educativa chilena. Educação y Sociedade, 32, 305-322.

Bang, C. (2014). Estrategias comunitarias en promoción de salud mental: Construyendo una trama conceptual para el abordaje de problemáticas psicosociales complejas. Psicoperspectivas, 13(2), 109-120.

Bilbao, M., Techio, E., Zubieta, E., Cárdenas, M., Páez, D., Barrientos, J., Blanco, A. (2011). Bienestar subjetivo y psicológico-social: El impacto de la violencia colectiva. En Páez, Superando la violencia colectiva y construyendo cultura de paz. Madrid: Editorial Fundamentos. 
Blanco, A., y Díaz, D. (2005) El bienestar social: su concepto y medición. Psicothema, 17, 582-589.

Bustos, C. y Cornejo, R. (2014). Sentido del trabajo en docentes de aulas hospitalarias: las emociones y el presente como pilares del proceso de trabajo. Psicoperspectivas, 14(2), 186-197.

Casas, F. (1996). Bienestar social. Una introducción psicosociológica. Barcelona: PPU.

Certo, J. L., y Fox, J. E. (2002). Retaining quality teachers. The High School Journal, 86, 57-75.

Cornejo, R. (2007). Bienestar/malestar docente y condiciones de trabajo en profesores de enseñanza media de Santiago. Ministerio de Educación de Chile, Fondo Nacional de Investigación y Desarrollo en Educación - FONIDE, Santiago de Chile.

Cornejo, R. (2008). Salud laboral docente y condiciones de trabajo. Revista Docencia, 35, 77-85.

Darling-Hammond, L. (1995). Policy for restructuring. In A. Lieberman (Ed.), The work of re-structuring schools: Building from the ground up (p. 137-152). New York: Teachers College Press.

Díaz, J., y Sánchez, M. P. (2002). Relaciones entre estilos de personalidad y satisfacción autopercibida en diferentes áreas vitales. Psicothema, 14, 100-105.

Diener, E. (1994). El bienestar subjetivo. Intervención Psicosocial, 3, 67-113.

Diener, E., Suh, M., Lucas, E., y Smith, H. (1999). Subjective well-being. Three decade of progress. Psychological Bulletin, 125, 276-302.

Donoso, S. (2004). Reforma político educacional en Chile 1990 -2004: El neoliberalismo en crisis. Estudios Pedagógicos, 1, 113 -135.

Ewing, R.A., y Smith, D. L. (2003). Retaining quality beginning teachers in the profession. English Teaching: Practice and Critique, 2, 15-32.

Feldfeber, M. (2006). Reforma educativa y regulación estatal. Los docentes y las paradojas de la autonomía impulsada por decreto. En M. Feldfeber y Andrade, D. (Eds.). Politicas educativas y trabajo docente. Nuevas regulaciones, Nuevos sujetos. Buenos Aires: Novedades Educativas.

Gil, F., y Alcover de la Hera, J. (1999). Introducción a la psicología de los grupos. Madrid: Psicología Pirámide.

Keyes, C. (1998). Social well-being. Social Psychology Quarterly, 61, 121-140.

Ley de Subvención Preferencial (s/f). Extraído desde http://www.educacion2020.cl/index.php?option=com_docmanytask.
Laca Arocena, F., Santana Aguilar, H., Ochoa Madrigal, Y., y Mejía Ceballos, J. (2011). Percepciones de bienestar social, anomia, interés e impotencia política en relación con las actitudes hacia la democracia. Liberabit. Revista de Psicología, 17, 7-17.

López, V. y Ascorra, P (2012). Miradas a la violencia en el espacio social de la escuela. Psicoperspectivas, 11(2), 1-7.

Núñez, I. (2002). La formación de docentes. Notas históricas. En B. Ávalos (Ed.). Profesores para Chile: Historia de un Proyecto (p. 14-39). Santiago: Ministerio de Educación.

Páez, D., Beristain, C., González-Castro, J. L., Basabe, N., y de Rivera, J. (2011). Superando la violencia colectiva y construyendo cultura de paz. España: Editorial Fundamentos.

Park, N., Peterson, C. H., y Sun, J. (2013). La psicología positiva: Investigación y aplicación. Terapia Psicológica, 1, 11-19.

Pfeffer, J. (1983). Organizational demography. En Cummings, L. y Staw, B. M. (Eds.). Research in organizational behavior (pp. 299-357). Greenwich, CT: JAI Press.

Pillay, H., Goddard, R., y Wills, L. (2005). Well-being, burnout and competence: Implications for teachers. Australian Journal of Teacher Education, 30, 22-33.

Prieto, M. (2001). Mejorando la calidad de la Educación. En M. Prieto (Ed.). La escuela: Una comunidad que aprende. Valparaíso: Ediciones Universidad Católica de Valparaíso.

Redondo, J. (2002). La salud laboral docente en el contexto de los requerimientos actuales en educación. Docencia, 18, 52-67.

Robalino, M. (2005). Actor o protagonista: Dilemas y responsabilidades sociales de la profesión docente. Revista Prelac, 1, 7-23.

Singh, K., y Billingsley, B. S. (1996). Intent to stay in teaching. Remedial and Special Education, 17, 37-48.

Sisto, V., y Fardella, C. (2011). Nuevas políticas públicas, epocalismo e identidad: El caso de las políticas orientadas a los docentes en Chile. Revista de Estudios Universitarios, 37, 123-141.

Unesco (2005). Condiciones de trabajo y salud docente: Un estudio de caso de Chile, Uruguay, Argentina, México, Ecuador y Perú. Santiago: Oficina Regional de Educación de la UNESCO para América Latina y el Caribe, OREALC / UNESCO.

Véliz-Burgos, A. (2012). Propiedades psicométricas de la escala de bienestar psicológico y su estructura factorial en universitarios chilenos. Psicoperspectivas 11(2), 143-163. 
